Original Paper http://ajol.info/index.php/ijbcs http://indexmedicus.afro.who.int

\title{
Analyse technico-économique des Aliments densifies sur les performances de croissances des boucs roux de Maradi au Niger
}

\author{
Mahamadou MALAM ABDOU ${ }^{1 *}$, Salissou ISSA ${ }^{1}$, Abdou. DAN GOMMA ${ }^{1}$ et \\ Germain Jérome SAWADOGO ${ }^{2}$
}

\author{
${ }^{1}$ Institut National de Recherches Agronomiques du Niger, BP 429 Niamey-Niger. \\ ${ }^{2}$ Ecole Inter-états des Sciences et Médecine Vétérinaires - BP 5077 Dakar - Fann-Dakar, Sénégal. \\ *Auteur correspondant ; E-mail : mamalam1@yahoo.fr ; Tél : (+227) 96888675 , (+227) 90011322
}

\section{RESUME}

Une étude portant sur l'influence des aliments densifiés sur la performance de croissance des boucs roux de Maradi a été conduite entre juillet 2016 à septembre 2016. Quarante deux (42) boucs ont été répartis au hasard en six lots de sept individus. Chaque lot correspond à un aliment ou traitement préparé à partir des ingrédients broyés et les individus représentent les répétitions. Les animaux sont logés dans des cages individuelles. La période d'adaptation et de collecte des données étaient respectivement de 14 et 56 jours. Cinq aliments (T1, T2, T3, T4, T5) qui se distinguaient sur la base du type du fourrage grossier et de son pourcentage entrant dans la formulation, étaient comparés à un aliment témoin (T0) préparé à partir de la formulation paysanne. Les résultats obtenus indiquaient que T0, T3 et T1 étaient les meilleurs avec des GMQ respectifs de 78,3 g/j, 51,8 g/j et 50,9 g/j. Les indices de consommation (IC) sont de 7,1 pour T0, 10,3 pour T3 et 10,6 pour T1. Sur le plan économique, T0 offre un bénéfice net de 1373 FCFA par animal suivi de T1 avec 1316 FCFA par animal et T3 avec 1311 FCFA par animal. Cette expérience montre bien que les tiges de sorgho peuvent concurrencer les fanes d'arachide ou de niébé une fois qu'elles sont améliorées, dans l'alimentation des caprins.

(C) 2017 International Formulae Group. All rights reserved.

Mots clés : Aliments densifiés, performance, indice de consommation, bénéfice net, bouc roux de Maradi, Niger.

\section{Technico-economic analysis of densified feed on the growth performance of red bucks of Maradi (Niger Republic)}

\begin{abstract}
A study of influence of densified feed on the growth performance of red buck of Maradi was conducted from July to September 2016. Forty-two (42) red bucks were divided randomly into six blocks of seven buks. Each block corresponds to a diet formulated using crushed ingredients and the individual buck is considered as replication. The animals were allocated to individual pen. The adaptation period and data collection were 14 and 56 days respectively. Five treatments namely T1, T2, T3, T4 and T5 differed basis on type and proportion of roughages, were compared with the control (T0) which is the farmers' formulation. The treatments T0, T3
\end{abstract}


and T1 gave the best daily live weight gain of 78.3, 51.8 and $50.9 \mathrm{~g}$ respectively. The feed consumption index (FCI) were 7.1, 10.3 and 10.6 for T0, T3 and T1 respectively. Economically, T0 gave net profit of 1373 FCFA per animal followed by T1 and T3 with 1316 and 1311 FCFA respectively. This experiment showed that improved sorghum stover could replace groundnut and cowpea haulms in goats feeding.

(C) 2017 International Formulae Group. All rights reserved.

Keywords: Densified feed, performance, feed consumption index, net profit, red bucks of Maradi, Niger Republic.

\section{INTRODUCTION}

L'élevage des petits ruminants en général et des caprins en particulier est très fréquent dans le système agricole africain. $\mathrm{Ce}$ cheptel est en constante croissance avec $14,5 \%$ et $17,5 \%$ respectivement pour les ovins et les caprins (Tchouamo et al., 2005). En Afrique de l'Ouest l'élevage caprin est numériquement l'un des plus importants avec $37,2 \%$ du cheptel caprin continental (Missohou et al., 2016). Au Niger, les caprins ont atteint 14,3 millions de têtes soit $36,3 \%$ de l'effectif total du cheptel national qui était de 39 millions de têtes toutes espèces confondues (sauf la volaille) en 2013 (INS, 2014). La région de Maradi occupait la $2^{\text {ème }}$ place après celle de Zinder en effectif caprin avec 2,7 millions de têtes soit $18,7 \%$ de l'effectif total du pays (INS, 2014). Dans cette région $85 \%$ $\mathrm{du}$ cheptel caprin sont dans la zone agricole et conduits en élevage sédentaire. En milieu rural, la chèvre est présente même dans les ménages les plus démunis où elle est détenue par la femme. Selon Kouamo et al. (2015) la chèvre constitue une carte de crédit qui sert à faire face à un certain nombre de besoin quotidien comme les rites coutumiers, les mariages, les baptêmes, elle représente également une source d'aliment laitier pour la population rurale. Dans la zone Sud du Niger et de Maradi en particulier la chèvre rousse de Maradi est la race dominante des caprins où elle est largement métissée avec la chèvre du sahel qui occupe préférentiellement la partie Nord du pays (Marichatou et al., 2002).
L'importance de la chèvre rousse est bien connue pour sa prolificité (Marichatou et al., 2002), sa production viande avec un rendement à l'abattage qui varie de 40 à $50 \%$ et une bonne production laitière $(0,5$ à 1,5 $\mathrm{kg} / \mathrm{jour}$ ) au cours de la saison humide (Nouhou, 2014). La peau de la chèvre rousse est fine, souple et d'une solidité remarquable, c'est pourquoi, elle est très recherchée en maroquinerie de luxe (Maïga et al., 2008). Plusieurs actions nationales en faveur de sa diffusion en milieu paysan sont prises par l'Etat pour lutter contre la pauvreté et améliorer le revenu des ménages vulnérables. Dans la zone agricole de la région de Maradi, pendant la saison sèche (octobre à juin), les petits ruminants en général et les caprins en particulier sont conduits au pâturage pendant la journée et sont ramenés au village dans la soirée (Abdoul Habou et al., 2016). Au pâturage, les animaux divaguent dans les champs, le fourrage qu'ils rencontrent est constitué de reliquats de résidus de culture, de la paille des adventices développées dans les champs pendant la saison des pluies et du fourrage des ligneux (feuilles et gousses des acacias, feuilles des arbustes divers etc.). Au fur et à mesure que la saison avance, ce pâturage devient maigre et les animaux n'arrivent plus à couvrir leur besoin. Pendant la saison des pluies (juillet à septembre) où tous les champs sont occupés par les cultures, on note une insuffisance des aires de pâturage. Les petits ruminants dont les caprins sont claustrés. Ils sont attachés aux piquets à 
domicile et nourris d'herbe verte fauchée et du fourrage des ligneux (Piliostigma reticulatum, Sclérocaria birrea, Guiera senegalensis etc.). Dans ces conditions, l'insuffisance en quantité et en qualité du fourrage constitue une des principales contraintes de l'élevage des petits ruminants dans la zone. C'est pourquoi, il a été initié la présente activité dont l'objectif est de trouver une ou des formules alimentaires performantes et rentables, concourant à l'amélioration de la productivité des petits ruminants dont la production viande en saison sèche comme en saison pluvieuse. La présente étude consiste alors à évaluer la valeur nutritive des aliments densifiés préparés à base des fourrages de tiges de sorgho, fanes de niébé et d'arachide d'une part et d'autre part les performances de croissance des boucs en embouche et procéder au calcul de la rentabilité économique des différentes formules alimentaires.

\section{MATERIEL ET METHODES}

\section{Matériel}

\section{Milieu d'étude}

Cette étude a été conduite entre juillet 2016 à septembre 2016 au Centre Secondaire d'Elevage des Caprins de Maradi (CSECM) du Niger. C'est un centre de sélection et de diffusion de la chèvre rousse de Maradi. Créé en 1963, il a une superficie de 1850 ha. Il a pour coordonnées géographique $7^{\circ} 41^{\prime}$ de longitude Est et $13^{\circ} 43^{\prime}$ de latitude Nord et est localisé à 3,5 km à l'Est de la ville de Maradi. Le climat de la zone est du type tropical sec caractérisé par une saison pluvieuse allant de juin à septembre et une saison sèche d'octobre à mai. La pluviométrie annuelle varie de 400 à $600 \mathrm{~mm}$ et les températures $\mathrm{mini} / \mathrm{max}$ journalières oscillent entre $22{ }^{\circ} \mathrm{C} / 36{ }^{\circ} \mathrm{C}$ mais peuvent atteindre $29{ }^{\circ} \mathrm{C} / 43{ }^{\circ} \mathrm{C}$ au cours des mois d'avril et mai (Abdou et al., 2011; Morou et al., 2016).

\section{Animaux et habitat}

$\mathrm{Au}$ total, 42 boucs de race "chèvre rousse de Maradi » et pesant 13 à $17 \mathrm{~kg}$ ont été achetés sur les marchés de la commune de Djirataoua. Au démarrage, les boucs ont été identifiés par des boucles, déparasités à l'albendazole et vaccinés contre la peste des petits ruminants (PPR) et la pasteurellose. Ils ont reçu de plus, un complexe multi vitaminé injectable par période de 14 jours tout le long de l'essai qui a duré 70 jours dont 14 jours de phase d'adaptation et 56 jours de collecte de données. L'habitat est un hangar fait d'un mur d'une hauteur de $1 \mathrm{~m}$ surmonté de grillage. Le toit est en matériaux locaux (paille, séko et plastique) soutenus par des charpentes en bois. Le sol est une dalle, compartimentée en cages de $1 \mathrm{~m}$ x $2 \mathrm{~m}$ où les animaux sont attachés aux cordes et logés individuellement. Chaque matin les cages, les mangeoires et les abreuvoirs sont nettoyés avant de servir les animaux.

\section{Aliments}

Les ingrédients utilisés pour préparer les aliments sont broyés et mélangés selon la formulation du Tableau 1.

\section{Méthodes}

\section{Constitution des lots expérimentaux}

Les animaux étaient répartis de façon aléatoire dans les cages en six lots ou traitements comprenant sept individus. Chaque traitement correspondait à un aliment et chaque individu constitue une répétition d'un traitement donné. Les sept boucs représentant les répétitions d'un traitement sont répartis au hasard.

\section{Alimentation}

Chaque bouc était soumis à un des traitements, et recevait chaque jour, dans une mangeoire, deux à trois repas de $500 \mathrm{~g}$ par aliment. Les services se faisaient dans les intervalles de temps suivants : 9 à $10 \mathrm{~h}$ pour le 
premier repas, le deuxième entre $13 \mathrm{~h}$ à $14 \mathrm{~h}$ et le dernier entre $17 \mathrm{~h}$ à $18 \mathrm{~h}$ pour ceux qui ont vidé leurs mangeoires. L'eau de boisson était servie dans des abreuvoirs et renouvelée chaque matin entre $11 \mathrm{~h}$ et $12 \mathrm{~h}$.

\section{Collecte des données}

- Pesée des refus, des animaux et du fumier

Les refus des aliments étaient collectés et pesés chaque jour par animal. Par contre, pour ne pas stresser les animaux quotidiennement, la pesée des boucs se passait chaque matin du $14^{\text {ème }}$ jour avant le premier service du jour. La pesée des boucs au premier jour de collecte de données était considérée comme le poids initial. La production du fumier pour chaque bouc était également peser. Ainsi, à chaque $15^{\text {ème }}$ jour, la production du fumier du $14^{\text {ème }}$ jour était collectée et pesée. L'aliment, le refus et le fumier étaient pesés à l'aide d'un peson électronique de portée $20 \mathrm{~kg}$ avec une sensibilité de $10 \mathrm{~g}$, alors que les boucs étaient pesés à l'aide d'un peson de portée $100 \mathrm{~kg}$ et de sensibilité 100 g. Pour peser les boucs, l'une des extrémités du peson était suspendue à un trépied et à l'autre extrémité, un berceau où serait placé le bouc à peser. Au total deux fiches de collecte de données ont été conçues: une fiche de pesée des refus ou du fumier, et une autre pour la pesée des boucs.

\section{* Analyse des ingrédients et des} aliments

Les ingrédients intervenant dans la composition des aliments, les aliments préparés et le fumier étaient prélevés. Les échantillons des ingrédients et des aliments ainsi prélevés étaient soumis à l'analyse de la composition chimique auprès du Laboratoire d'Alimentation et de Nutrition Animale
(LANA) de l'INRAN. La caractérisation chimique de ces échantillons consistait à la détermination de la matière sèche $(\mathrm{MS})$, la matière minérale $(\mathrm{MM})$, la cellulose brute (CB), la matière azotée totale (MAT) ou protéines et les éléments phosphore $(\mathrm{P})$ et calcium (Ca).

\section{* Données économiques}

Les prix des ingrédients ont été collectés suite à une enquête menée en juillet, août et septembre de l'année 2015, sur les marchés de la commune de Djirataoua. La méthode consistait à peser l'unité locale de vente du produit pour avoir le poids, ensuite demander son prix de vente (Tableau 2). Pour les boucs, les prix d'achat et de vente étaient fixés par un intermédiaire. Les ingrédients intervenant dans la préparation des aliments, ont été broyés à l'aide d'un appareil du type C-DARMA - INRAN. Selon la fiche technique, ce broyeur a une capacité de 500 $\mathrm{kg}$ de fourrage broyé en 2 heures avec une consommation de 1 litre qui coûte 538 FCFA. Le coût de la main d'œuvre (2000 FCFA/j) utilisée pour les différents travaux (broyage des ingrédients, préparation des aliments et entretien des animaux) était le montant payé à un journalier adulte qui travaille pendant $6 \mathrm{~h}$. Pour le prix du fumier nous avons considéré le prix de $40 \mathrm{FCFA} / \mathrm{kg}$ de la ferme avicole de Maradi.

\section{Analyse statistique}

L'analyse de variance (ANOVA) à un facteur (les traitements) des données a été faite avec le logiciel IBM SPSS statistics 20 selon la procédure General Linear Model (GLM) et la comparaison des moyennes a été faite selon le test de Duncan au seuil de 5\%. 
Tableau 1: Formulation des aliments.

\begin{tabular}{lcccccc}
\hline Ingrédients & ${ }^{*}$ F0 & F1 & F2 & F3 & F4 & F5 \\
\hline Fanes d'arachide (\%) & 42 & 0 & 50 & 0 & 0 & 25 \\
Cosse de niébé (\%) & 20 & 0 & 0 & 0 & 0 & 0 \\
Grain de sorgho (\%) & 16 & 0 & 0 & 0 & 0 & 0 \\
Fanes de niébé (\%) & 0 & 50 & 0 & 0 & 25 & 0 \\
Tiges de sorgho (\%) & 0 & 0 & 0 & 50 & 25 & 25 \\
Gousses de Piliostigma reticulatum (\%) & 0 & 20 & 20 & 20 & 20 & 20 \\
Son de blé (\%) & 21 & 12 & 12 & 12 & 12 & 12 \\
Tourteau de coton (\%) & 0 & 10 & 10 & 10 & 10 & 10 \\
Calcaire de Malbaza (\%) & 0 & 3 & 3 & 3 & 3 & 3 \\
Phosphate Naturel de Tahoua (PNT) (\%) & 0 & 3 & 3 & 3 & 3 & 3 \\
Sel (\%) & 1 & 2 & 2 & 2 & 2 & 2 \\
\hline Total (\%) & 100 & 100 & 100 & 100 & 100 & 100 \\
\hline
\end{tabular}

(*) F0 est la formule paysanne de l'aliment à l'embouche (source enquête juin 2016).

Tableau 2: Prix moyen des différents ingrédients de l'alimentation des boucs.

\begin{tabular}{lcc}
\hline Ingrédients & Prix moyen (FCFA/kg) & Ecart type \\
\hline Fanes d'arachide & 83 & 5,72 \\
Sorgho grain & 145 & 4,11 \\
Fanes de niébé & 100 & 22,4 \\
son du blé & 120 & 2,83 \\
Cosses de niébé & 276 & 8,01 \\
Tiges de sorgho & 58 & 4,97 \\
Tourteau de grain de coton & 164 & 0 \\
Gousse de P.reticulatum & 35 & 5,12 \\
Calcaire de Malbaza & 60 & 0 \\
Phosphate naturel de Tahoua (PNT) & 60 & 0 \\
Sel de cuisine & 150 & 8,16 \\
\hline
\end{tabular}




\section{RESULTATS}

\section{Composition chimique des ingrédients et des aliments}

La composition chimique des ingrédients utilisés pour la préparation des aliments est présentée dans le Tableau 3. Ces ingrédients ont un taux en matière sèche (MS) qui tourne autour de $90 \%$ et celui des minéraux (calcaire de Malbaza et le phosphate naturel de Tahoua) est de $100 \%$. Le taux en matière minérale (MM) est inférieur à $10 \%$ allant de $2 \%$ pour le sorgho grain à $8 \%$ pour les cosses de niébé. Pour la cellulose brute $(\mathrm{CB})$, sa teneur est très faible dans les grains du sorgho avec une valeur de $5 \%$, elle est faible dans le son du blé avec une valeur de $11 \%$, moyenne dans les gousses de $P$. reticulatum avec $21 \%$ et relativement élevée dans les autres ingrédients avec des valeurs proche autour de $32 \%$. Par contre pour les matières azotées totales (MAT) ou protéines brutes, deux ingrédients se révèlent être plus riches: il s'agit du tourteau des grains de coton avec une valeur de $23,9 \%$ et le son de blé avec 16,9\%. Les autres ingrédients ont des valeurs variant de 7 à $9 \%$. Pour la teneur en phosphore, elle était très importante dans le tourteau de grain de coton avec $17,5 \mathrm{mg} / \mathrm{kg}$ de MS, un peu moins dans les gousses de $P$. reticulatum et les grains de sorgho avec 14,2 mg/kg de MS pour le premier et $13 \mathrm{mg} / \mathrm{kg}$ de MS pour le second. Le son du blé, les fanes d'arachide et de niébé ont des taux proches en phosphore qui tournent autour de $10 \mathrm{mg} / \mathrm{kg}$ de MS, tandis que les cosses de niébé et les tiges de sorgho sont pauvres avec 5,5 $\mathrm{mg} / \mathrm{kg}$ de MS. S'agissant du calcium (Ca), les ingrédients utilisés sont pauvres en calcium avec des valeurs inférieures à $5 \mathrm{mg} / \mathrm{kg}$ de MS sauf pour les cosses de niébé, les fanes d'arachide et de niébé qui ont des taux proches de 10 $\mathrm{mg} / \mathrm{kg}$ de MS et le calcaire de Malbaza, contient $118 \mathrm{mg} / \mathrm{kg}$ de MS de Ca.
Pour les aliments, d'une manière générale, les six aliments préparés (T0, T1, T2, T3, T4 et T5) étaient similaires dans leur composition en matière sèche (MS), en matière minérale $(\mathrm{MM})$ et en cellulose brute (CB) sauf pour l'aliment T3 qui affichait une valeur légèrement faible (6\%) de $\mathrm{MM}$ (Tableau 4). S'agissant du taux en MAT, il était plus élevé au niveau de l'aliment $\mathrm{T} 1$ avec $12,2 \%$ suivi de $\mathrm{T} 2$ avec une valeur de 10,7\%. Les aliments T0, T4 etT5 avaient une valeur identique de 9,7 \% de MAT et l'aliment T3 avec une valeur plus faible de $8,6 \%$. Pour le phosphore (P) les six aliments avaient des valeurs proches comprises entre $16,2 \mathrm{mg} / \mathrm{kg}$ de MS pour T0 et $19,8 \mathrm{mg} / \mathrm{kg}$ de MS pour T2. Alors que pour le calcium (Ca) les aliments, $\mathrm{T} 3$ et $\mathrm{T} 5$ étaient les moins riches avec des valeurs respectives de $13 \mathrm{mg} / \mathrm{kg}$ de MS et $14 \mathrm{mg} / \mathrm{kg}$ de MS. Les autres aliments avaient des valeurs de $16 \mathrm{mg} / \mathrm{kg}$ de MS pour T0 et T2, $19 \mathrm{mg} / \mathrm{kg}$ de MS pour T4 et 20 $\mathrm{mg} / \mathrm{kg}$ de MS pour T1.

\section{Performance de l'ingestion de la matière sèche et de croissance pondérale des boucs}

Au démarrage de l'essai, les poids des boucs étaient comparables $(\mathrm{P}>0,05)$ entre les traitements. Ces poids variaient de $13,5 \mathrm{~kg}$ pour le traitement T5 à $14,9 \mathrm{~kg}$ pour le traitement T4. Il en est de même à la fin de l'essai qui a duré 56 jours, les poids des animaux étaient comparables $(\mathrm{P}>0,05)$ entre les traitements avec $15,9 \mathrm{~kg}$ pour T5 et 18,2 $\mathrm{kg}$ pour $\mathrm{T} 0$. Les quantités de matières sèches totales ingérées par bouc durant l'essai étaient comparables $(\mathrm{P}>0,05)$ également entre les traitements. Ces quantités variaient de $25,2 \mathrm{~kg}$ pour T5 à $30,8 \mathrm{~kg}$ pour T2. Les quantités moyennes de matière sèches ingérées (MSI) par jour par bouc ont varié de $449,5 \mathrm{~g} / \mathrm{j}$ pour l'aliment $\mathrm{T} 5$ à $549,2 \mathrm{~g} / \mathrm{j}$ pour l'aliment T2. Converties en $\mathrm{g} / \mathrm{j} / \mathrm{kg}$ de poids métabolique $\left(\mathrm{g} / \mathrm{j} / \mathrm{kgP}^{0,75}\right)$, ces quantités consommées ont évolué de $60,2 \mathrm{~g} / \mathrm{j} / \mathrm{kgP}^{0,75}$ 
pour T5 à $69,1 \mathrm{~g} / \mathrm{j} / \mathrm{kgP}^{0,75}$ pour T2 (Tableau 5). Exprimées en $\mathrm{g} / \mathrm{j}$ ou en $\mathrm{g} / \mathrm{j} / \mathrm{kgP}^{0,75}$ les quantités de MS ingérées ne sont pas significativement différentes $(\mathrm{P}<0,05)$ entre les aliments cela montre une indifférence des animaux dans la consommation des six aliments fournis. Par contre, les gains de poids vif et les GMQ étaient très significativement différents $(\mathrm{P}<0,05)$ entre les aliments. En effet le GMQ pour l'aliment T0 est le plus élevé (78,3 g/j), suivi de ceux des aliments $\mathrm{T} 3$ et $\mathrm{T} 1$ avec des valeurs respectives de $51,8 \mathrm{~g} / \mathrm{j}$ et $50,9 \mathrm{~g} / \mathrm{j}$. Les GMQ étaient faibles pour T2 $(44,6 \mathrm{~g} / \mathrm{j})$ et $\mathrm{T} 5(41,1$ $\mathrm{g} / \mathrm{j})$ et très faible pour l'aliment $\mathrm{T} 4(29,8 \mathrm{~g} / \mathrm{j})$. Le gain de poids vif, d'une valeur de $4,4 \mathrm{~kg}$ pour l'aliment T0 était élevé par rapport à ceux de T1 et T3 qui sont identiques avec une valeur de 2,9 kg. Ceux de T2 et T5 étaient comparables avec $2,5 \mathrm{~kg}$ pour $\mathrm{T} 2$ et 2,4 $\mathrm{kg}$ pour T5. Le gain de poids vif le plus faible enregistré est celui de T4 avec une valeur de 1,7 kg (Tableau 5). En outre, il est ressorti dans ce tableau une différence significative $(\mathrm{P}<0,05)$ de l'Indice de Consommation (IC). L'indice de Consommation (IC) qui traduit l'efficacité de transformation de l'aliment en viande (quantité de matière sèche consommée en $\mathrm{kg}$ pour gagner $1 \mathrm{~kg}$ de poids vif) était meilleur pour les aliments T0, T3, T1et T5 avec des valeurs respectives de $7,1,10,3,10,6$ et 11,5 et mauvais pour l'aliment $\mathrm{T} 2$ et $\mathrm{T} 4$ avec des valeurs de 14,5 et 21,5 .

\section{Evaluation économique de production}

Le coût de production d'un kilogramme de chaque aliment préparé, a été déterminé sur la base des coûts des ingrédients selon leurs proportions dans la formulation de l'aliment. Ce coût est plus élevé pour la formule paysanne comparé aux autres formules et plus faible au niveau de T3, ceci est surtout lié au faible prix aux tiges de sorgho par rapport aux fanes d'arachide ou de niébé. Par contre, les frais liés au broyage des ingrédients sont les plus faibles pour l'aliment T0, car à ce niveau, seules les fanes d'arachides sont broyées; les cosses de niébé et les grains de sorgho sont utilisés à l'état nature. Au niveau des autres aliments, la différence des frais liés au broyage est surtout liée à la quantité d'aliment consommée par l'animal. Pour déterminer les charges liées à la production d'un bouc par aliment, en plus du prix d'achat de l'animal, il faut ajouter, le coût de l'aliment consommé par l'animal, le coût lié au soin sanitaire et celui de la main d'œuvre pour la préparation de l'aliment, l'hygiène et le gardiennage de l'animal pendant toute la période de l'essai (Tableau 6). Dans l'ensemble, On remarquait que, hormis les frais de l'acquisition de l'animal, c'était l'alimentation qui occupait plus de $50 \%$ des charges suivie de la main d'œuvre $(20 \%$ à $30 \%)$ pour la production d'un animal au niveau de tous les traitements.

$\mathrm{Au}$ niveau du Tableau 7, il apparait que les recettes relevaient seulement de la vente des boucs et du fumier produits par ces derniers à la fin de l'essai. Le bénéfice brut par bouc obtenu est alors la différence entre la recette et la charge liées à chaque bouc au niveau du traitement. Au niveau de cet essai seul le broyeur est amorti, ainsi le tableau 7 montrait que le bénéfice net par bouc décroissait de l'aliment $\mathrm{T} 0, \mathrm{~T} 1, \mathrm{~T} 3, \mathrm{~T} 5$ à $\mathrm{T} 2$ avec respectivement 1373 F CFA, 1316 FCFA, 1311 FCFA, 678 et 459 FCFA. Pour l'aliment T4 il a été enregistré une perte de 162 FCFA qui peut être dû à la faible performance de croissance observée chez les boucs nourris avec cet aliment. 
Tableau 3: Composition chimique des ingrédients de l'alimentation des boucs.

\begin{tabular}{lcccccc}
\hline & & \multicolumn{3}{c}{$\%$ de MS } & & Ca \\
\cline { 3 - 6 } Ingrédients & M.S (\%) & MM & CB & MAT & $\begin{array}{c}\text { P (mg/kg } \\
\text { de MS) }\end{array}$ & $\begin{array}{c}\text { (mg/kg } \\
\text { de MS) }\end{array}$ \\
\hline Fanes de niébé & $88 \pm 0,17$ & $7 \pm 0,55$ & $33 \pm 0,16$ & 8,8 & 9,8 & 9 \\
Cosse de niébé & $91 \pm 0,04$ & $8 \pm 0,12$ & $33 \pm 0,04$ & 7 & 5,5 & 8,6 \\
Tourteau de coton & $92 \pm 0,30$ & $5 \pm 0,05$ & $31 \pm 0,58$ & 23,9 & 17,5 & 1,4 \\
Tiges de sorgho & $90 \pm 0,26$ & $4 \pm 0,19$ & $31 \pm 0,30$ & 7,2 & 5,5 & 4 \\
Grains de sorgho & $90 \pm 0,05$ & $2 \pm 0,10$ & $5 \pm 0,20$ & 7,9 & 13 & 1,6 \\
Gousses de Piliostigma. & & & & & & \\
reticulatum & $89 \pm 0,11$ & $5 \pm 0,07$ & $21 \pm 0,75$ & 8,4 & 14,2 & 3 \\
Fanes d'arachide & $88 \pm 0,07$ & $7 \pm 0,22$ & $32 \pm 0,29$ & 9,3 & 10,5 & 10 \\
Son de blé & $91 \pm 0,03$ & $6 \pm 0,15$ & $11 \pm 0,30$ & 16,5 & 10,2 & 1,6 \\
Phosphate naturel de & & & & & & \\
Tahoua & $100 \pm 0,00$ & $95 \pm 0,00$ & 0 & 0 & 62,5 & 33 \\
Calcaire de Malbaza & $100 \pm 0,00$ & $68 \pm 0,00$ & 0 & 0 & 0 & 118 \\
\hline
\end{tabular}

Légende : $\mathrm{MS}=$ matière sèche, $\mathrm{MM}=$ matière minérale, $\mathrm{CB}=$ cellulose brute, $\mathrm{MAT}=$ matière azotée totale, $\mathrm{P}=$ phosphore, $\mathrm{Ca}=$ calcaire.

Tableau 3: Composition chimique des aliments formulés.

\begin{tabular}{|c|c|c|c|c|c|c|}
\hline \multirow[b]{2}{*}{ Aliments } & \multirow[b]{2}{*}{ M.S (\%) } & \multicolumn{3}{|c|}{$\%$ de $\mathbf{M S}$} & \multirow[b]{2}{*}{$P(\mathrm{mg} / \mathrm{kg}$ de $\mathrm{MS})$} & \multirow[b]{2}{*}{ Ca (mg/kg de MS) } \\
\hline & & MM & CB & MAT & & \\
\hline T0 & $91 \pm 0,26$ & $8 \pm 0,37$ & $31 \pm 0,34$ & 9,7 & 16,2 & 16 \\
\hline $\mathrm{T} 1$ & $92 \pm 0,24$ & $10 \pm 0,05$ & $32 \pm 0,14$ & 12,2 & 17,5 & 20 \\
\hline $\mathrm{T} 2$ & $92 \pm 0,44$ & $9 \pm 0,17$ & $34 \pm 0,28$ & 10,7 & 19,8 & 16 \\
\hline $\mathrm{T} 3$ & $90 \pm 0,02$ & $6 \pm 0,24$ & $31 \pm 0,17$ & 8,6 & 17,2 & 14 \\
\hline $\mathrm{T} 4$ & $92 \pm 0,27$ & $11 \pm 0,03$ & $35 \pm 0,28$ & 9,7 & 18,5 & 19 \\
\hline $\mathrm{T} 5$ & $92 \pm 0,02$ & $11 \pm 0,43$ & $32 \pm 0,14$ & 9,7 & 17,5 & 13 \\
\hline
\end{tabular}


Tableau 4: Performance de l'ingestion de la matière sèche et de la croissance pondérale des boucs selon les aliments.

\begin{tabular}{|c|c|c|c|c|c|c|c|c|}
\hline Paramètres & T0 & T1 & T2 & T3 & T4 & T5 & ESM & $\mathbf{P}_{\text {value }}$ \\
\hline$P_{\text {initial }}(\mathrm{kg})$ & $13,8^{\mathrm{a}}$ & $13,7^{\mathrm{a}}$ & $14,5^{\mathrm{a}}$ & $14,3^{\mathrm{a}}$ & $14,9^{\mathrm{a}}$ & $13,5^{\mathrm{a}}$ & 0,76 & 0,774 \\
\hline$P_{\text {final }}(\mathrm{kg})$ & $18,2^{\mathrm{a}}$ & $16,5^{\mathrm{a}}$ & $17,1^{\mathrm{a}}$ & $17,1^{\mathrm{a}}$ & $16,8^{\mathrm{a}}$ & $15,9^{\mathrm{a}}$ & 0,88 & 0,582 \\
\hline Gain de poids vif $(\mathrm{kg})$ & $4,4^{\mathrm{a}}$ & $2,9^{\mathrm{b}}$ & $2,5^{\mathrm{ab}}$ & $2,9^{\mathrm{b}}$ & $1,7^{\mathrm{c}}$ & $2,4^{\mathrm{ab}}$ & 0,37 & 0,001 \\
\hline Gain de poids moyen (GMQ) $(\mathrm{g} / \mathrm{j})$ & $78,3^{\mathrm{a}}$ & $50,9^{\mathrm{b}}$ & $44,6^{\mathrm{bc}}$ & $51,8^{\mathrm{b}}$ & $29,8^{\mathrm{c}}$ & $41,1^{\text {bc }}$ & 6,57 & 0,001 \\
\hline Matière sèche ingérée totale (kg) & $30,1^{\mathrm{a}}$ & $29,6^{\mathrm{a}}$ & $30,8^{a}$ & $28,6^{\mathrm{a}}$ & $27,5^{\mathrm{a}}$ & $25,2^{\mathrm{a}}$ & 2,10 & 0,471 \\
\hline Matière sèche ingérée $(\mathrm{g} / \mathrm{j})$ & $536,5^{\mathrm{a}}$ & $527,6^{\mathrm{a}}$ & $549,2^{\mathrm{a}}$ & $509,7^{\mathrm{a}}$ & $491,4^{\mathrm{a}}$ & $449,5^{\mathrm{a}}$ & 37,48 & 0,474 \\
\hline Matière sèche ingérée totale $\left(\mathrm{g} / \mathrm{j} / \mathrm{kg} \mathrm{P}{ }^{0,75}\right)$ & $67,3^{\mathrm{a}}$ & $68,8^{\mathrm{a}}$ & $69,1^{\mathrm{a}}$ & $64,2^{a}$ & $62,4^{\mathrm{a}}$ & $60,2^{\mathrm{a}}$ & 3,61 & 0,419 \\
\hline IC (Kg de MSI/kg de Gain de poids vif) & $7,1^{\mathrm{a}}$ & $10,6^{\mathrm{a}}$ & $14,5^{\mathrm{ab}}$ & $10,3^{\mathrm{a}}$ & $21,5^{\mathrm{b}}$ & $11,5^{\mathrm{a}}$ & 2,92 & 0,030 \\
\hline
\end{tabular}

Tableau 5: Charge de production d'un bouc en fonction des différentes formulations alimentaires.

\section{Paramètres}

Prix d'achat des animaux (F CFA /bouc) (a)

Coût de l'aliment (F CFA/kg) (b)

Quantité moyenne d'aliment consommée par bouc $(\mathrm{kg})$ (c)

Coût de carburant de broyage des ingrédients (F CFA) (d)

Coût moyen de l'aliment d'un bouc (FCFA) (e) $=(b * c)+(d)$

Gain de poids vif réalisé $(\mathrm{kg})$

Coût alimentaire du kg de gain de poids vif (F CFA)

Coût moyen des produits vétérinaires /bouc (F CFA) (f)

(*)Coût de main d'œuvre (F CFA) (g)

Charges totales de production par bouc $(\mathrm{F} \mathrm{CFA})(\mathbf{1})=(\mathrm{a})$

$\begin{array}{llllll}\text { T0 } & \text { T1 } & \text { T2 } & \text { T3 } & \text { T4 } & \text { T5 }\end{array}$

$\begin{array}{llllll}16615 & 16495 & 17458 & 17097 & 17940 & 16254\end{array}$

$\begin{array}{llllll}140 & 94 & 86 & 73 & 84 & 79\end{array}$

$\begin{array}{llllll}33 & 32 & 33,4 & 31,7 & 29,9 & 27,4\end{array}$

$\begin{array}{llllll}15 & 28 & 29 & 28 & 26 & 24\end{array}$

$\begin{array}{llllll}4635 & 3036 & 2901 & 2342 & 2538 & 2189\end{array}$

$\begin{array}{lllll}4,4 & 2,9 & 2,5 & 2,9 & 1,7\end{array} 2,4$

$1053,4 \quad 1046,9 \quad 1160,6 \quad 807,6 \quad 1492,7 \quad 911,9$

$\begin{array}{llllll}595 & 595 & 595 & 595 & 595 & 595\end{array}$

$\begin{array}{llllll}1120 & 1120 & 1120 & 1120 & 1120 & 1120\end{array}$

$\begin{array}{llllll}22965 & 21246 & 22074 & 21154 & 22193 & 20158\end{array}$


Tableau 6: Bénéfice par bouc en fonction des différentes formulations alimentaires.

\begin{tabular}{|c|c|c|c|c|c|c|}
\hline Paramètres & T0 & T1 & T2 & T3 & T4 & T5 \\
\hline Prix de vente des animaux (FCFA/bouc (2) & 23912 & 21935 & 21905 & 21905 & 21393 & 20368 \\
\hline $\begin{array}{l}\text { Production moyenne du fumier /bouc/ en } 56 \mathrm{j} \\
(\mathrm{kg})\end{array}$ & 14,9 & 19,9 & 20 & 18,3 & 20,2 & 16 \\
\hline $\begin{array}{l}\text { (*)Prix de vente du fumier produit/bouc (F } \\
\text { CFA) (3) }\end{array}$ & 598 & 799 & 800 & 732 & 810 & 640 \\
\hline Recette par bouc $($ FCFA) $(4)=(2)+(3)$ & 24510 & 22734 & 22705 & 22637 & 22203 & 21008 \\
\hline Bénéfice brut $(\mathrm{F} \mathrm{CFA})(\mathbf{5})=(4)-(1)$ & 1545 & 1488 & 631 & 1483 & 10 & 850 \\
\hline Amortissement équipement (broyeur) (6) & 172 & 172 & 172 & 172 & 172 & 172 \\
\hline Bénéfice net (FCFA) (5) - (6) & 1373 & 1316 & 459 & 1311 & -162 & 678 \\
\hline
\end{tabular}

(*) : Le prix du fumier de $40 \mathrm{~F} / \mathrm{kg}$ est celui de la station avicole de Maradi (enquête juillet 2015).

\section{DISCUSSION}

Les taux de Matière sèche (MS) obtenus de l'analyse des ingrédients, variaient de $88 \%$ à $92 \%$ et $100 \%$. Ces taux sont légèrement inférieurs aux taux rapportés par Gnanda et al. (2005), Gnanda et al. ( 2015a) dont la valeur moyenne est de $93 \%$ qui sont eux sont même inférieurs à ceux rapportés par Kandombo et Nianogo (2001) avec des valeurs variant de $94,5 \%$ à $97,26 \%$. Quand aux pourcentages en matière minérale (MM) et en Matière azoté totale (MAT), ils sont similaires à ceux trouvés par Kandombo et Nianogo (2001), Gnanda et al. (2015a) avec des valeurs inférieures à $10 \%$ de MS, sauf au niveau des fanes d'arachide où ces deux auteurs ont trouvé un taux en MM de 13,72\% pour le premier et $18,82 \%$ pour le second. En revanche, le tourteau affiche dans les différents cas des valeurs importantes en MAT avec $23,9 \%$ pour notre étude, $29,72 \%$ pour Gnanda et al. (2015a) et jusqu'à 41,63\% pour Kandombo et Nianogo (2011).
Les aliments préparés ont des taux en matière sèche (MS) qui variaient de $88 \%$ à $92 \%$. Ces pourcentages sont légèrement inférieurs à ceux obtenus par Kiema et al. (2008), Gnanda et al. (2015a, 2015b) et Soumana et al. (2016) avec des ingrédients de même nature comme les tiges de sorgho, fanes de niébé, fanes d'arachides, cosse de niébé et tourteau de coton et les gousses de $P$. reticulatum. Par contre les pourcentages en MAT sont légèrement supérieurs aux pourcentages rapportés par Gnanda et al (2015b). Ils sont compris entre 8 à $12 \%$, ce qui est suffisant pour le bon fonctionnement de la microflore du rumen des petits ruminants. La quantité de MS volontairement ingérée n'a pas significativement variée entre les aliments, cela pourrait être dû à la similitude de la granulométrie des aliments servis. Les résultats enregistrés $(60,2 \mathrm{~g} / \mathrm{j} / \mathrm{kg} \mathrm{P}$ 0,75 à $69,2 \mathrm{~g} / \mathrm{j} / \mathrm{kg} \mathrm{P}^{0,75}$ ) sont comparables à ceux de Pamo et al., (2010) qui variaient de $63,5 \mathrm{~g} / \mathrm{j} / \mathrm{kg} \mathrm{P}^{0,75}$ à $81,1 \mathrm{~g} \mathrm{/j} / \mathrm{kg} \mathrm{P}^{0,75}$ sur les caprins au Cameroun supplémentés avec du 
Mucuna pruriens. Par contre ils sont inférieurs à ceux de Zoundi et al. (2005), Anigbogu et al. (2006), et Kiema et al. (2008) sur l'embouche ovine. En effet Zoundi et al. (2005) ont trouvés des chiffres variant de $70,98 \mathrm{~g} / \mathrm{j} / \mathrm{kg} \mathrm{P}^{0,75}$ à $94,47 \mathrm{~g} / \mathrm{j} / \mathrm{kg} \mathrm{P}^{0,75}$. Anigbogu et al. (2006) ont rapporté des valeurs de 75,2 à $86,7 \mathrm{~g}$ de $\mathrm{MS} / \mathrm{j} / \mathrm{kg} \mathrm{P} \mathrm{P}^{0,75}$ qui sont proches de ceux de Kiema et al., (2008) compris entre 71,7 à $89,8 \mathrm{~g} / \mathrm{j} / \mathrm{kg} \mathrm{P}{ }^{0,75}$. La faible ingestion des aliments peut s'expliquer par la manière dont les boucs choisissaient ce qu'ils désiraient ingérer. En effet, ce comportement est très favorable sur parcours puisqu'il permettait à l'animal de sélectionner les fractions végétales les plus nutritives qu'il pouvait rencontrer.

Le GMQ qui traduit la vitesse de croissance pondérale variait de 29,8 à $78,3 \mathrm{~g} / \mathrm{j}$ et était supérieur à celui rapporté par Pamo et al., (2010) dont les valeurs variaient entre $10,2 \mathrm{~g} / \mathrm{j}$ à $18,3 \mathrm{~g} / \mathrm{j}$. Nos résultats étaient semblables à ceux de Gnanda et al. (2015a). Ils étaient également comparables à ceux de Molid Aziada (2016) au niveau de trois de ses traitements donnant des GMQs de 59; 75 ; et $79 \mathrm{~g} / \mathrm{j}$; mais inférieurs aux deux autres qui ont des valeurs de 95 et118 g/j. Les GMQs trouvés dans cette étude sont nettement inférieur à ceux obtenus par Anigbogu et al. (2006) (116 à $162 \mathrm{~g} / \mathrm{j})$ et Kiema et al. (2008) $(139,53 \mathrm{~g} / \mathrm{j}$ à $162,06 \mathrm{~g} / \mathrm{j})$, portant sur l'incorporation d'amandes de Mangifera indica séchées dans les rations concentrées d'agneaux en croissance pour le premier auteur et sur la valorisation des ressources locales dans l'embouche ovine pour le second.

A l'exception des aliments $\mathrm{T} 2$ et $\mathrm{T} 4$, les indices de consommation (IC) de T0, T1, $\mathrm{T} 3$ et $\mathrm{T} 5$ étaient comparables aux indices de consommation (IC) notés par Zoundi et al. (2005), Kiema et al., (2008), de Kondombo et Nianogo (2001) ou de Molid Aziada (2016) qui ont mené des études sur l'alimentation des ovins en embouche avec des ingrédients locaux. Cela veut dire que les caprins ont la même efficacité sinon plus, que les ovins de transformation d'une quantité de MS en 1 kilogramme de viande.

$\mathrm{Du}$ point de vue performance économique de l'essai, il ressortait que les bénéfices nets étaient globalement positifs et variaient de 459 à 1373 FCFA par bouc sauf pour l'aliment $\mathrm{T} 4$ avec lequel on enregistrait une perte de devise de -162 FCFA par bouc. Les bénéfices nets obtenus étaient nettement inférieurs à ceux mentionnés par plusieurs auteurs (Zoundi et al., 2002, 2005 ; Gnanda et al., 2005 ; Ayantunde et al., 2008 ; Kiema et al., 2008 ; Molid Aziada, 2016) qui ont tous travaillé sur l'embouche ovine pourtant les indices de consommations (IC) trouvés sur les caprins étaient comparables à ceux obtenus avec les ovins. Les meilleurs aliments étaient T0 suivi de T1 et T3. Les tiges de sorgho une fois améliorée pouvaient nourrir les caprins en élevage intensif en permettant à l'éleveur d'avoir un profit.

\section{Conclusion}

Les résultats obtenus de cette étude montre que la formule paysanne est plus bénéfique que les autres formules. Cependant, l'alimentation avec les tiges de sorgho broyées semble être intéressante pour les caprins du fait qu'elle engendre un bénéfice semblable à celui de l'alimentation à base de fanes de niébé ou d'arachide. En plus, le bénéfice net dégagé par animal n'était pas très élevé même pour les aliments les plus performants (T0 suivi de $\mathrm{T} 1$ et $\mathrm{T} 3$ ), cela peut être lié aux prix des ingrédients et le prix des animaux pendant la période expérimentale. Ces bénéfices nets faibles peuvent expliquer aussi les raisons pour lesquelles les producteurs ne s'adonnent pas à l'embouche des caprins même s'ils ont un IC comparable sinon plus intéressant que celui des ovins. Toutefois ces aliments préparés permettent l'entretien des caprins même pendant la saison des pluies où ils sont gardés à domicile et nourris à l'auge pendant trois à quatre mois. 


\section{CONFLIT D'INTERETS}

Les auteurs de ce manuscrit déclarent qu'il n'y a aucun conflit d'intérêt entre eux.

\section{CONTRIBUTIONS DES AUTEURS}

Dans la présente étude, MMA a assuré l'élaboration du protocole de recherche, la collecte, le traitement des données et la rédaction du manuscrit sous l'encadrement de SI. ADG a contribué à la relecture des différentes versions pour l'amélioration de la qualité scientifique du manucrit et GJS a donné des conseils tout le long du processus.

\section{REMERCIEMENTS}

Les auteurs adressent leurs sincères remerciements au Centre National de Spécialisation en Elevage (CNS-El), à l'INRAN et au Centre Secondaire d'Elevage de Caprins de Maradi (CSEM) pour les moyens matériels et financiers consentis à la réalisation de cette étude.

\section{REFERENCES}

Abdou N, Nsahlaia IV, Chimonyoa M. 2011. Effects of groundnut haulms supplementation on millet stover intake, digestibility and growth performance of lambs. Animal Feed Science and Technology, 169: 176-184. Doi:10.1016/j.anifeedsci.2011.07.002

Abdoul Habou Z, Boubacar MK, Adam T. 2016. Les systèmes de productions agricoles du Niger face au changement climatique : défis et perspectives. Int. J. Biol. Chem. Sci., 10(3): 1262-1272. DOI: http://dx.doi.org/10.4314/ijbcs.v10i3.28

Anigbogu NM, Bienstman P, Van Damme B, Ezeokoli CD. 2006. Incorporation of Dry Mangifera indica Kernel in the Concentrate Ration of Growing Lambs. Revue Elev. Méd. Vét. Pays trop., 59(14) : $39-42$.

Ayantunde AA, Fernandez-Rivera S, DanGomma A. 2008. Sheep Fattening with Groundnut Haulms and Millet Bran in the West African Sahel. Revue Elev.
Méd. Vét. Pays Trop., 2008, 61(3-4): 215-220.

Gnanda IB, Bougouma-Yameogo MCV, Wereme N'diaye A, Ouedraogo $\mathrm{T}$, Kabore A, Lodoun B, Sinon B. 2015b. L'embouche bovine dans les élevages du Plateau Central du Burkina Faso : Résultats économiques d'une démarche de validation d'un référentiel technicoéconomique sur la spéculation. Int. $J$. Biol. Chem. Sci., 9(6): 2648-2662. DOI : http://dx.doi.org/10.4314/ijbcs.v9i6.

Gnanda IB, Nianogo AJ, Zoundi JS, Somda J, Koanda S. 2005. Performances techniques et économiques de l'embouche ovine en exploitation traditionnelle de la région sahélienne au Burkina Faso. Sciences et Médecine, Rev. CAMES - Série A, (3) : 49 - 56.

Gnanda IB, Nignan M, Ouedraogo S, Wereme N'diaye A, Traore O, Sinon B. 2015a. Influence d'une co-construction de rationnement amélioré sur les performances d'embouche ovine paysanne dans la commune rurale de Korsimoro au Burkina Faso. Int. J. Biol. Chem. Sci., 9(3): 1544-1556. DOI : http://dx.doi.org/10.4314/ijbcs.v9i3.35

Institut National de Statistique (INS) 2014. Annuaire Statistique 2009 - 2013, Ministère du plan, de l'aménagement du territoire et du développement communautaire, République du Niger, $245 \mathrm{p}$.

Kiema A, Nianogo AJ, Ouedraogo T, Somda J. 2008. Valorisation des ressources alimentaires locales dans l'embouche ovine paysanne: performances technicoéconomiques et option de diffusion. Cahiers Agricultures, 17(1) : 23 - 27.

Kondombo SR, Nianogo AJ. 2001. Performance d'ovins Djallonké alimentés à base de résidus de récolte au Burkina Faso. Agronomie Africaine, 13(2) : $59-66$.

Kouamo J, Mpatswenumugabo JP, Sow A, Kalandi M, Sawadogo GJ. 2015. 
Efficacité d'un traitement combiné à base d'acétate de fluorogestonecloprosténol-eCG sur l'induction de l'oestrus et la fertilité des chèvres sahéliennes. Revue Méd. Vét. Pays Trop., 166(5-6) : 163-169.

Maïga AM, Diane I, Mazou I, Djibrillou A. 2008. Etude sur la compétitivité des filières viande rouge / cuirs et peaux. Rapport final, PRODEX / MDA, République du Niger, p. 104.

Marichatou H, Mamane L, Banoin M, Baril G. 2002. Performances zootechniques des caprins au Niger : étude comparative de la chèvre rousse de Maradi et de la chèvre à robe noire dans la zone de Maradi. Revue Elev. Méd. Vét. Pays Trop., 55(1) : $79-84$.

Missohou A, Nahimana G, Ayssiwede SB, Sembene M. 2016. Elevage caprin en Afrique de l'Ouest: une synthèse. Rev. Elev. Méd. Vét. Pays Trop., 69 (1) : 3-18.

Molid Aziada M. 2016. Evaluation technique et économique des formules alimentaires de Blocs Multi Nutritionnels en embouche ovine au Niger. Mémoire de Master PADD / IPA, EISMV, Université Cheikh Anta Diop de Dakar, p.28.

Morou B, Ounani H, Amadou Oumani A, Diouf A, Guero C, Mahamane A. 2016. Caractérisation de la structure démographique des ligneux dans les parcs agroforestiers du terroir de Dan Saga (Aguié, Niger). Int. J. Biol. Chem. Sci., 10(3): 1295-1311. DOI : http://dx.doi.org/10.4314/ijbcs.v10i3.31

Nouhou EM. 2014. Effet de substitution de tourteau de graines de coton par les gousses de Piliostigma reticulatum (De Candolle) Hochstetter dans l'alimentation sur les performances de croissance de la chèvre rousse de Maradi, Mémoire Master PADD/IPA, EISM, Dakar, p. 40.

Pamo TE, Ko Awono PMD, Tendonkeng F, Ngo Tama AC, Boukila B. 2010. Performances de croissance des caprins supplémentés avec Mucuna pruriens au Nord Cameroun. Revue Elev.Méd.Vét. Pays Trop., 63 (1-2) : 47 - 52.

Soumana I, Ayssiwede SB, Issa S, Guero, Missohou A. 2016. Effet de la complémentation avec des blocs multinutritionnels concentrés à base de Sida cordifolia $L$., une plante invasive, sur les performances zootechnico-économiques des antenais de race Balami à Déréké/Dosso (Niger). International Journal of Innovation and Applied Studies, $\quad \mathbf{1 5}(3)$ : $\quad$ 716-730. http://www.ijias.issr-journals.org/

Tchouamo IR, Tchoumboué J, Thibault L. 2005. Caractéristiques socio économiques et techniques de l'élevage des petits ruminants dans la province de l'Ouest du Cameroun. Tropicultura, 23(4): 201 - 211.

Zoundi JS, Nianogo AJ, Sawadogo L. 2002. Utilisation optimale de ressources alimentaires localement disponibles pour l'engraissement des ovins au sein des exploitations mixtes agriculture-élevage $\mathrm{du}$ plateau central du Burkina Faso. Revue Elev. Méd. Vét. Pays Trop., 55(1) : $53-62$.

Zoundi SJ, Sawadogo L, Nianogo JA. 2005. Utilisation de blocs multi-nutritionnels en substitution partielle de concentré pour l'engraissement des ovins au sein des systèmes mixtes agriculture élevage du plateau central du Burkina Faso. Journal des Sciences, 5(1): 15-27. 